\title{
Congenital Pulmonary Airway Malformation in One of the Monochorionic Diamniotic Twins - A Rare Encounter
}

\author{
Rohan Kumar Singh ${ }^{1}$, Akhita Singhania², Gaurav Vedprakash Mishra ${ }^{3}$, Prerna Anup Patwa ${ }^{4}$ \\ 1, 2, 3, 4 Department of Radiodiagnosis, Acharya Vinoba Bhave Rural Hospital, DMIMS, \\ Sawangi (Meghe), Wardha, Maharashtra, India.
}

\section{INTRODUCTION}

Congenital Pulmonary Airway Malformation (CPAM) is a fetal pulmonary development abnormality caused by airway dysgenesis. It is characterized by cystic or adenomatous lesions in terminal bronchioles. Severity of a case is prognosed by size of mass, degree of mediastinal shift and presence of hydrops and polyhydramnios. CPAM being a rare pathology, is often accidentally diagnosed during routine antenatal ultrasounds. Congenital pulmonary airway malformation in the past was known as Congenital Cystic Adenomatoid Malformation (CCAM) as it is a congenital disorder of lung just similar to broncho pulmonary sequestration. Usually an entire lobe of lung is replaced by a non-working cystic area of abnormal lung tissue in this condition and this abnormal tissue never functions as normal lung tissue. In this case report we present a distinct case of CPAM which belonged to Type I of Stocker's classification in one of the two monochorionic diamniotic twins, grayscale ultrasound findings are discussed here.

Cause for congenital pulmonary airway malformation is still unknown. Its incidence is approximately 1 in every 30000 pregnancies. Malignant transformation can occur within cyst with small documented risk. Hence, early diagnosis and surgical resection is necessary to prevent grave complications. Congenital pulmonary airway malformation is also known as congenital cystic degeneration which is a rare abnormality of fetal lung development. In this disease there is a benign cystic or adenomatous lung tumour which grows on terminal bronchioles and restricts their function. According to various research data, incidence of CCAMs is reportedly between 1:8300 and 1:35000 live births. ${ }^{1}$ This congenital pulmonary airway malformation mainly affects males but still there is no evidence that sex has any effect on probability of development of this disease in individuals. ${ }^{2}$ Based on clinical and pathological features it is classified into 5 major types. Most CPAM lesions are manageable with the proper assessment, diagnosis and surgical interventions. ${ }^{3}$
Corresponding Author: Dr. Prerna Anup Patwa, Department of Radiodiagnosis, Acharya Vinoba Bhave Rural Hospital, DMIMS, Sawangi (Meghe), Wardha, Maharashtra,

India.

E-mail: prernapatwa17@gmail.com

DOI: $10.14260 / \mathrm{jemds} / 2020 / 810$

How to Cite This Article:

Singh RK, Singhania A, Mishra GV, et al. Congenital pulmonary airway malformation in one of the monochorionic diamniotic twins - a rare encounter. J Evolution Med Dent Sci 2020;9(48):36943696, DOI: $10.14260 /$ jemds/2020/810

Submission 18-08-2020,

Peer Review 15-10-2020,

Acceptance 21-10-2020,

Published 30-11-2020.

Copyright (c) 2020 Rohan Kumar Singh et al. This is an open access article distributed under Creative Commons Attribution License [Attribution 4.0 International (CC BY 4.0)]

\section{PRESENTATION OF CASE}

A 20-year-old pregnant female patient came to the Emergency Department of Acharya Vinoba Bhave Rural Hospital, Sawangi, Meghe, with complaints of generalized weakness and pain in both upper and lower limbs of one day duration. Patient with her first twin pregnancy was seen for prenatal care and her first ultrasound at 14.5 weeks of gestation. One of female monochorionic diamniotic twins was diagnosed with congenital pulmonary airway malformation type I of Stocker's classification of right lung. An enlarged right lung with single large cyst of size $12 \mathrm{x}$ $10 \mathrm{~mm}$ (Figure 1, 2, 3; Coronal, axial and sagittal section respectively), a compressed left lung, a compressed and displaced heart were observed while the other fetus was having no abnormality. 

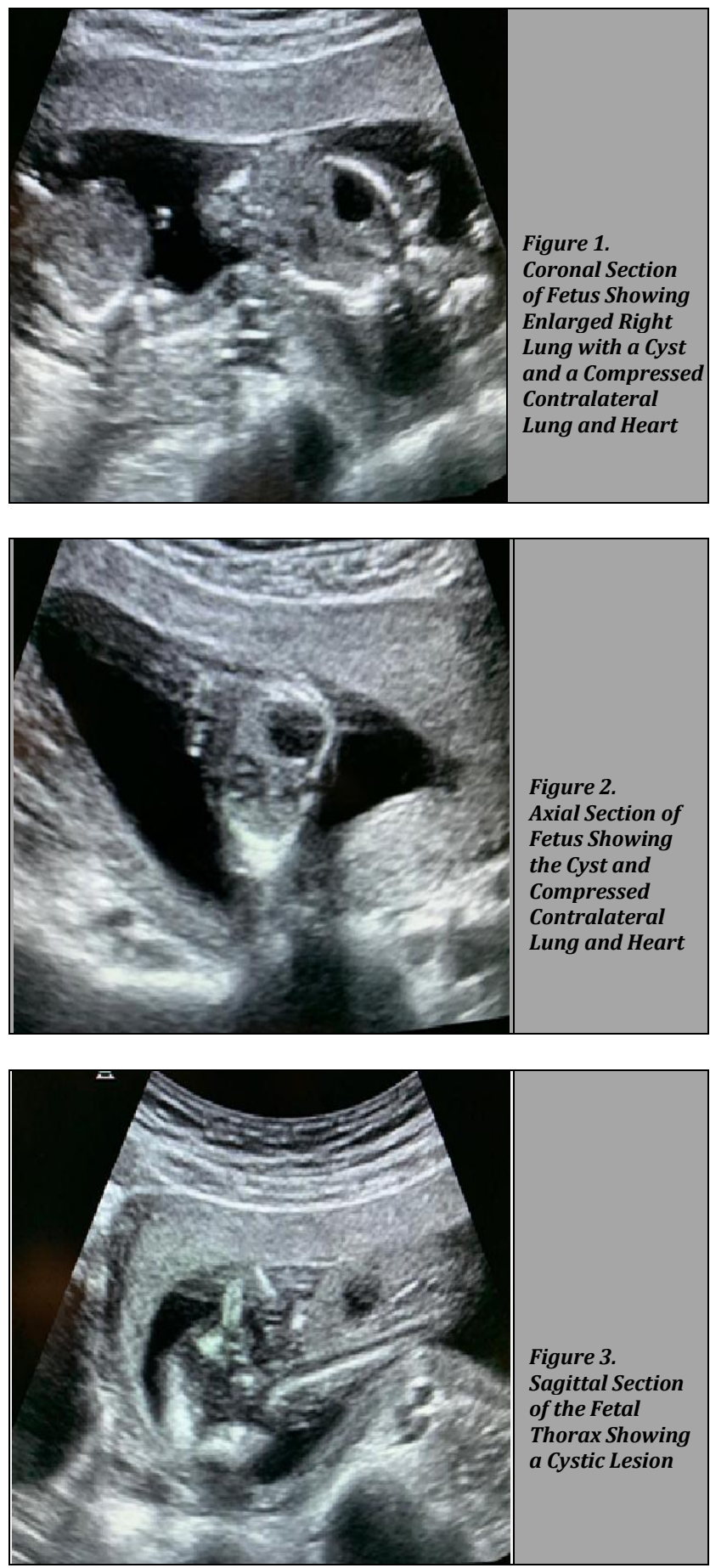

\section{DISCUSSION}

CPAM is a rare condition distinguished by immature lung which is malformed and has cystic appearance. New-borns and still born infants are affected by CPAM, and unusually children beyond infancy are known to be affected. It is a hamartomatous lesion that is usually symptomatic in first few days of life. The babies with CPAM present as neonates with severe, progressive respiratory distress due to cyst expansions. Hydrops may be present ${ }^{4}$. Exact aetiology of CPAM is not known, although it is considered to be as hamartomatous malformation and abnormal proliferation of pulmonary tissue at different sites.

Sometimes CPAM may communicate proximally with airways, although this form is abnormal. Most CPAMs are supplied by the pulmonary artery and their drainage is by the pulmonary veins, except hybrid lesions which have a systemic blood supply. Fetal ultrasound in large cyst CPAM will show multiple variable-sized anechoic spaces with echogenic soft tissue. On fetal MR (Magnetic Resonance) imaging, large cyst CPAM manifests as hyperintense unilocular or multilocular cysts with well-defined walls on T2-weighted images. On postnatal chest radiography variable density in the region of the mass with mediastinal shift is seen. In neonatal period, a large cyst CPAM is seen as a round soft-tissue mass gradually filled with air, with delayed clearance of fetal lung fluid from those cysts through abnormal airway. Air-fluid levels may be visualized. There may be a solitary well-defined air-filled cyst with imperceptible walls or multiple cysts of varying size. On gross pathological examination of an excised large cyst CPAM we can appreciate a large $(>2 \mathrm{~cm})$ cyst and occasionally multiple or multiloculated cysts, often with small cysts that will be merging with the adjacent lung. Microscopically, the cysts have ciliated epithelium as their lining, and may contain mucin forming epithelium as well. This highlights the embryologic origin of the lung-foregut derivative. ${ }^{5}$ On prenatal ultrasound, a small cyst CPAM is an echogenic mass with multiple small cysts whereas a microcystic (predominantly solid-appearing) CPAM is homogeneously echogenic. There may be mediastinal shift and a consequent polyhydramnios and hydrops in fast-growing CPAM. The indicators of poor prognosis large lesions, bilateral lung involvement, and hydrops.

It has been proposed that designation of this lesion "CCAM" be changed to "CPAM" to reflect the fact that lesions described as cystic are present in only 3 of the 5 types and "Adenomatoid" only in one type (Type I). CPAM is classified as follows. ${ }^{6}$

- CPAM Type 0 - Acinar dysplasia / agenesis is rare malformation largely incompatible with life. Lungs are small, firm with diffusely granular surface.

- CPAM Type I - It accounts for nearly $65 \%$ of cases. It is operable with good prognosis. Grossly, lesion is predominantly cystic type (measuring $3-10 \mathrm{~cm}$ in diameter) surrounded by smaller cysts.

- CPAM Type II - accounts for $10 \%-15 \%$ of cases and mainly seen in first year of life. It has poor prognosis because it is frequently associated with other congenital anomalies. Grossly lesion is composed of medium sized cysts measuring 0.5 to $2.0 \mathrm{~cm}$ in diameter that are evenly distributed and blend with the adjacent normal parenchyma. CPAM Type II has been noted in nearly $50 \%$ cases of extra-lobar sequestration.

- CPAM Type III - It is infrequent and accounts only about $5 \%$ of cases. It consists of small cystic or solid type exclusively seen in first few days to months of life with characteristic male preponderance. It is seen to be associated with maternal polyhydramnios, fetal anasarca commonly. So it has high mortality rate. Also, this is increased alfa-fetoprotein levels in the second trimester. Grossly cysts are small measuring less than $0.2 \mathrm{~cm}$ in diameter, affecting large bulky mass involving an entire lobe or even an entire lung.

- CPAM Type IV - It is hamartomatous malformation of distal acinus and accounts for $10 \%-15 \%$ of cases with 
an age range of new-born to 4 years. This lesion involves a single lobe. 5

It's peculiar to note that the infants who are diagnosed to have CPAM lesions on antenatal ultrasonography are later found to be asymptomatic at birth. Most of these CPAM are associated with other congenital anomalies. ${ }^{7}$ Congenital lobar emphysema can be differentiated from CPAM as it contains broncho vascular marking which extends to the periphery. In certain cases, when a macrocystic form of CPAM appears, there is a risk of pulmonary hypoplasia and development of fetal hydrops due to compression of developing lungs by large cysts ${ }^{8}$. Fetuses suffering from CPAM can have various survivability prognoses and it depends on following factors: size of growth, degree of mediastinal displacement, hydrops and polyhydramnios. Good, moderate, and poor prognosis groups can be identified based on these factors. However, as number of studies examining CPAM is growing around world, it is being discovered that many cystic growths regress on their own in the third trimester of pregnancy. ${ }^{8}$

Expectant management is useful for non-hydropic fetus, although the survival of fetuses with hydrops may improves only with fetal intervention. If the mass is predominantly a single cyst, thoracoamniotic shunting may be an option. After 32 weeks of gestation, surgical resection of the mass may be possible while the fetus is maintained on placental circulation, using an Ex Utero Intrapartum Treatment ("EXIT") procedure. If the condition is not recognized antenatally on ultrasound, it is often detected when a baby of less than 2 years of age presents as respiratory difficulty or a subsequent pulmonary infection. Symptomatic infants are treated with surgical resection, where lobectomy or segmental resection is done. ${ }^{5}$

\section{CONCLUSIONS}

CPAM basically is a benign cystic or adenomatous terminal bronchiole disorder which is caused by a respiratory tract malformation. Its early diagnosis is based on ultrasound examination of the pregnant woman. Most of the CPAM lesions can be managed with the proper assessment, diagnosis and surgical interventions.

Financial or other competing interests: None.

Disclosure forms provided by the authors are available with the full text of this article at jemds.com.

\section{REFERENCES}

[1] Gornall AS, Budd JLS, Draper ES, et al. Congenital cystic adenomatoid malformation: accuracy of prenatal diagnosis, prevalence and outcome in a general population. Prenatal Diagnosis: Published in Affiliation With the International Society for Prenatal Diagnosis 2003;23(12):997-1002.

[2] Baird R, Puligandla PS, Laberge JM. Congenital lung malformations: informing best practice. Seminars in Pediatric Surgery 2014;23(5):270-7.

[3] Sirithangkul S, Chuengchitraks S, Staworn D, et al. Late manifestation of congenital cystic adenomatoid malformation with lung abscess: a case report. J Med Assoc Thai 2010;93(Suppl 6):223-7.

[4] Chan IC, Lee YS, Tsao PC, et al. Congenital pulmonary airway malformation type 4: a case report. J Pediatr Resp Dis 2013;9:48-52.

[5] Biyyam DR, Chapman T, Ferguson MR, et al. Congenital lung abnormalities: embryologic features, prenatal diagnosis and postnatal radiologic-pathologic correlation. Radiographics 2010;30(6):1721-38.

[6] Stocker JT, Dehner LP. Pediatric pathology. $2^{\text {nd }}$ edn. Philadelphia: Lippincott Williams \& Wilkins 2001: p. 46673.

[7] Sittig SE, Asay GF. Congenital cystic adenomatoid malformation in the newborn: two case studies and review of the literature. Respiratory Care 2000;45(10):1188-95.

[8] Goldstein RB, Callen PW. Ultrasound evaluation of the fetal thorax and abdomen. Ultrasonography in obstetrics and gynecology. $2^{\text {nd }}$ edn. Philadelphia: WB Saunders 1988;207:439-42. 